\title{
Model of flare lightcurve profile observed in soft $\mathrm{X}$-rays
}

\author{
Magdalena Gryciuk ${ }^{1,2}$, Marek Siarkowski ${ }^{1}$, Szymon Gburek ${ }^{1}$, \\ Piotr Podgorski ${ }^{1}$, Janusz Sylwester ${ }^{1}$, Anna Kepa ${ }^{1}$ \\ and Tomasz Mrozek ${ }^{1,2}$ \\ ${ }^{1}$ Solar Physics Division, Space Research Centre Polish Academy of Sciences, \\ Kopernika 11, 51-622 Wroclaw, Poland \\ email: mg@cbk.pan.wroc.pl \\ ${ }^{2}$ Astronomical Institute, University of Wroclaw, Poland
}

\begin{abstract}
We propose a new model for description of solar flare lightcurve profile observed in soft X-rays. The method assumes that single-peaked 'regular' flares seen in lightcurves can be fitted with the elementary time profile being a convolution of Gaussian and exponential functions. More complex, multi-peaked flares can be decomposed as a sum of elementary profiles. During flare lightcurve fitting process a linear background is determined as well. In our study we allow the background shape over the event to change linearly with time. Presented approach originally was dedicated to the soft X-ray small flares recorded by Polish spectrophotometer SphinX during the phase of very deep solar minimum of activity, between $23^{\text {rd }}$ and $24^{\text {th }}$ Solar Cycles. However, the method can and will be used to interpret the lightcurves as obtained by the other soft X-ray broad-band spectrometers at the time of both low and higher solar activity level. In the paper we introduce the model and present examples of fits to SphinX and GOES 1-8 $\AA$ channel observations as well.
\end{abstract}

Keywords. Sun: corona, flares, X-rays

\section{Introduction}

The most basic flare characteristics such as duration and its amplitude can be determined from the analysis of lightcurves in respective energy passbands. Because of statistical noise, data gaps and time-overlapped events, it is a challenge to obtain these properties in systematic and consistent way. The problem can be solved by using a mathematical formula for representation of simple flare time profile. The suitable model should be backed up by physical interpretation and be consistent with observations.

The studies of solar flares observed by Polish spectrometer SphinX (Solar Photometer in X-rays; Sylwester et al. (2012); Gburek et al. (2011a); Gburek et al. (2011b); Sylwester et al. (2011); Sylwester et al. (2012); Gburek et al. (2013) have revealed a need to define such profile applicable to the observations. The spectrophotometer measured soft X-ray (SXR) emission (within the energy range from 1.2 to $15 \mathrm{keV}$ ) from the entire solar hemisphere thus the obtained lightcurve contains many time-overlapped events. To analyse particular flares which can be observed in SphinX lightcurve we decided to invent and use a formula for the mathematical representation of so-called elementary soft X-ray flare time profile. Our goal was to parametrize a simple, single-peaked events and decompose complex, multi-peaked flares into a series of elementary profiles. Such decomposition allow to estimate parameters of individual flares independently.

The parametrisation of the flare lightcurves using mathematical functions have already been attempted by other authors. As an example, we mention the analysis by Aschwanden 
et al. (1998) where authors have shown that hard X-ray flare flux pulses observed by BATSE/CGRO (Compton Gamma Ray Observatory; Fishman et al. (1989)) can be fitted by logistic curve. Appropriate model for stellar white-light flare time profile of M type dwarf observed by Kepler space observatory (Borucki et al. (2010)) was presented by Davenport et al. (2014). Their method assumes different models of time profile for rise and decay flare phases as they assume a fourth-order polynomial and a sum of two exponential curves respectively.

Below we propose a new approach suitable for interpretation of soft X-ray solar flare lightcurve profile with possible extension to other energy bands in which flare lightcurves are observed. The model was originally invented for flares observed by SphinX, however, it is compatible with GOES 1-8 A flare observations as well. The model application and agreement with observed lightcurves are presented for example set of flares observed by both instruments.

\section{Elementary Flare Time Profile Definition}

In our approach we describe elementary soft X-ray flare temporal profile based on the assumption that SXR flares time profile should reflect two simultaneous processes, which are impulsive energy release (representing by gaussian energy deposition function, e.g. Aschwanden et al. 1998) and the process of energy losses (described by exponential function). Therefore the flare profile $f(t)$ is described by the following equation:

$$
f(t)=\int_{0}^{t} g(x) h(t-x) d x
$$

where

$$
\begin{gathered}
g(t)=A \exp \left(-(t-B)^{2} / C^{2}\right), \\
h(t)=\exp (-D t),
\end{gathered}
$$

where $A, B, C, D$ are the shape parameters and $t$ stays for time.

Equation can be converted to a form easier for numerical integration which reads:

$$
f(t)=\frac{1}{2} \sqrt{\pi} A C \exp \left(D(B-t)+\frac{C^{2} D^{2}}{4}\right) *\left(\operatorname{erf}(Z)-\operatorname{erf}\left(Z-\frac{t}{C}\right)\right),
$$

where

$$
Z=\frac{2 B+C^{2} D}{2 C}
$$

and $\operatorname{erf}$ is the error function, defined as $\operatorname{erf}(t)=\frac{2}{\sqrt{\pi}} \int_{0}^{t} \exp \left(-s^{2}\right) d s$. The error function is widely used in statistics and thus available in many programming languages for symbolic and numerical calculations. It is also available in IDL which was the main software environment used for the purposes of the present study.

For the background SXR emission on which the flare sits we assume linear model $(b(t)=E t+F)$ with two free parameters $E$ and $F$. Eventually our formulae to describe observed flare lightcurve $l c(t)$ reads

$$
l c(t)=f(t)+b(t)
$$

and depends on the value of the six parameters $(A, B, C, D$ - flare shape; $E, F$ - linear background). 


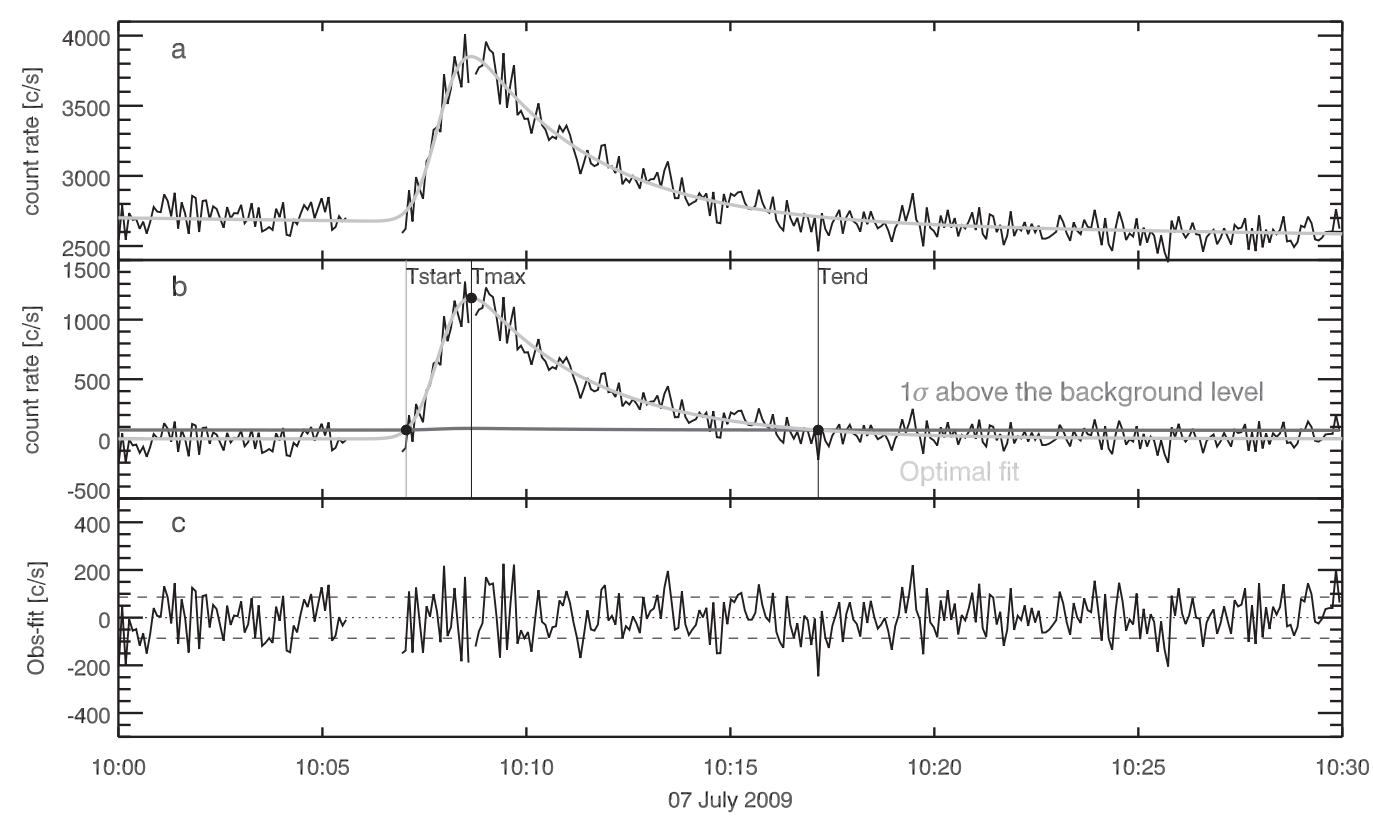

Figure 1. Example of SphinX lightcurve as observed in the energy range $1.2-15 \mathrm{keV}$ before (panel a) and after (panel b) background subtraction with the best quality fit of the elementary flare profile (grey curve). Times of the start, maximum and the end are marked with straight vertical lines. The horizontal line indicates a level of $1 \sigma$ above the background, which is used to the determination of flare start and end times. Panel c shows the residua. Levels of 0 and $\pm 1 \sigma$ of the residua are marked as dotted and dashed lines.

Term of 'elementary' in the context of temporal flare profiles was originally introduced by van Beek (van Beck et al. 1974) to describe hard X-ray flares structuring. Van Beek and co-authors found that hard X-ray flares can be decomposed into a number of smaller spikes, and called these spikes 'Elementary Flare Bursts'. In this work we use term 'elementary' for formula of simple flare time profile as seen in the soft X-rays where the most of flare thermal energy is concentrated.

For fitting $l c(t)$ to observed flares' profile identified in lightcurve we used IDL function MPFITFUN (Markwardt 2009), published and available through the webpage:

http : //www.physics.wisc.edu/ craigm/idl/mpfittut.htm. The MPFITFUN algorithm was translated from MINPACK-1 package (More 1977) for solving nonlinear equations and nonlinear least squares problems. We found MPFITFUN faster, more flexible and of improved performance in comparison to the generic routine CURVEFIT available within the IDL environment.

We use fitted flare profiles to determine more reliable estimates the time of event start, end end the maximum as well as its magnitude, i.e. the flare amplitude after background subtraction. Event start time was defined as the time when analysed flare profile based on shape parameters $(\mathrm{A}, \mathrm{B}, \mathrm{C}, \mathrm{D})$ raised above one standard deviation value $(1 \sigma)$ above the background. The end time was defined as the moment when fitted profile dropped below that level on the flare decay phase.

In Figure 1 we plot an example of flare lightcurve observed by SphinX on 07 July 2009 with the best quality fit to the function of the elementary profile defined by Equation 2.5. Flare maximum occured at 10:08:38 UT. Times of start, maximum and the end are marked with vertical lines in panel b. Panel c shows residua (data-fit) with the lines of values 0 and $\pm 1 \sigma$. 


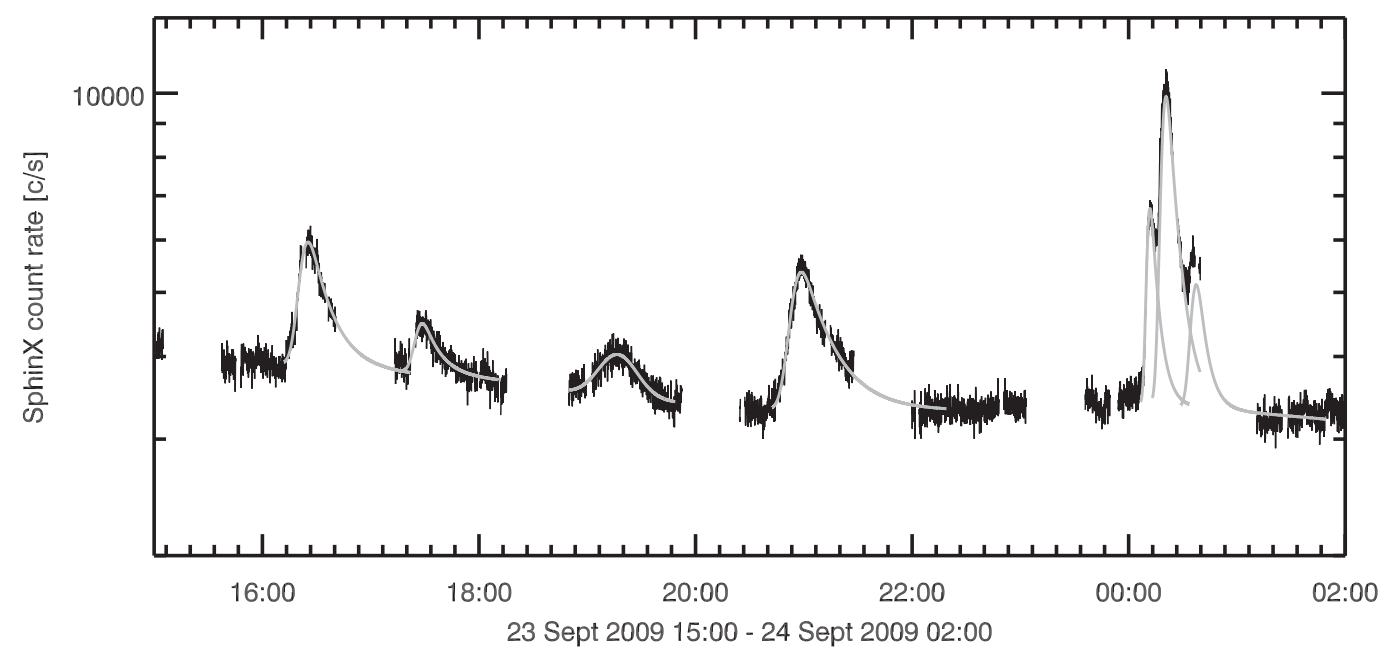

Figure 2. SphinX 1.2 - $15 \mathrm{keV}$ lightcurve with optimal fit of elementary flares (Equation 2.4). The last three events in the plot are examples of overlapped flare profiles. The gray lines represent the best quality fits to the individual events. The fitted profiles are plotted from the start to the end time of respective flares.

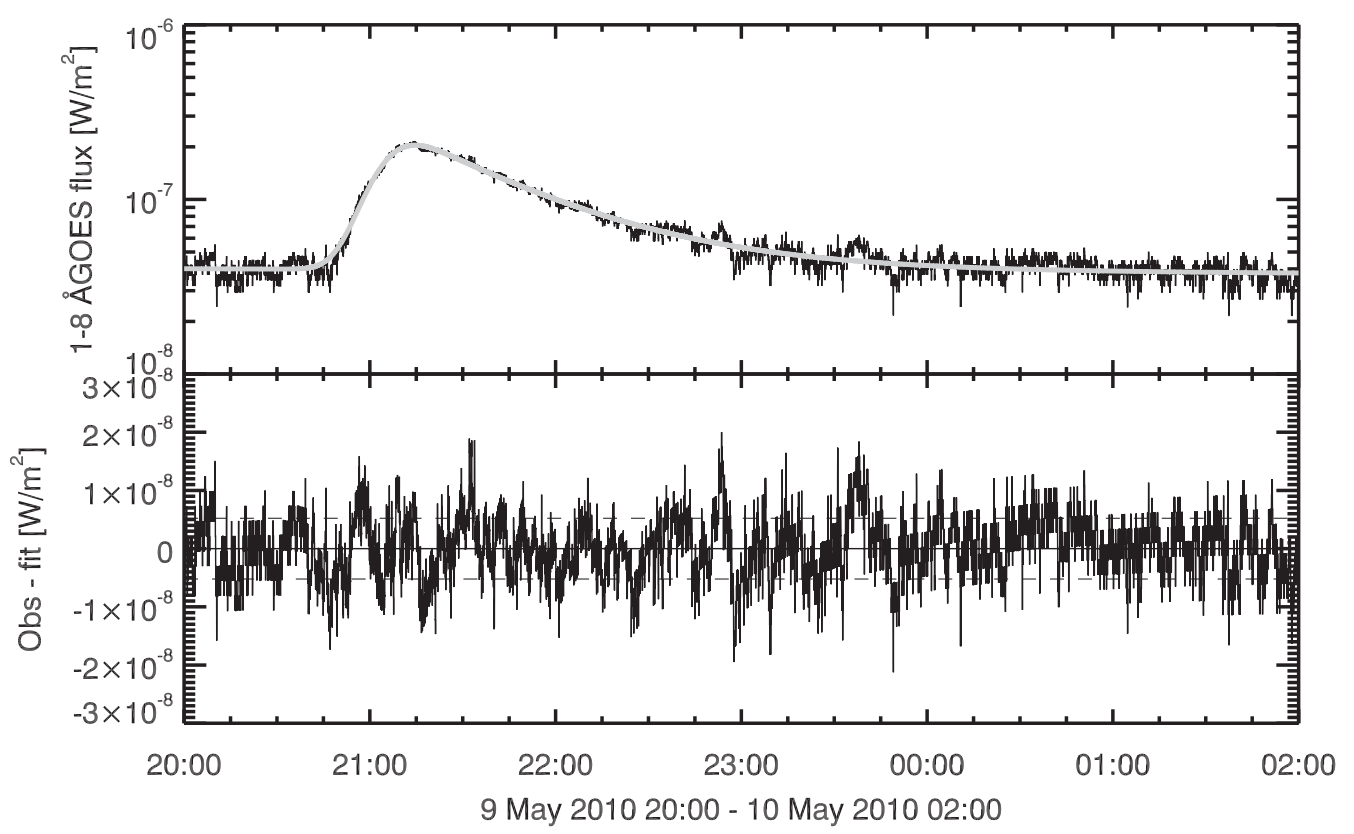

Figure 3. An example of GOES 1-8 A wavelength channel flare lightcurve with optimal fit of elementary flare time profile (gray line) described by Equation 2.4. The bottom plot represents residua, where levels of 0 and $\pm \sigma$ of the residua are marked as solid and dashed lines.

Our method also allows to fit complex multi-peaked profiles using series of individual single profiles given by the formula of elementary flare profile. Such profiles are observed when there are two or more flares overlapped in time. Figure 2 presents example lightcurve recorded by SphinX (black line) containing several consecutive flares observed on 23 - 24 September 2009 with the best quality fits of profile formula to particular events. The last three profiles in the plot represent an example of decomposition of events overlapped in 
time. Because of SphinX orbit passing through polar bands and South Atlantic Anomaly data gaps are present in analysed lightcurve.

Presented formula of elementary soft X-ray flare time profile can be successfully applied to the interpretation of flare observations by other SXR emission recording spectrophotometers as well. An example of fit to GOES 1-8 $\AA$ wavelength channel flare lightcurve is shown in Figure 3. The B 2.0 class flare occurred on 9-10 May 2010. Grey solid line represents best fit of formula of elementary flare time profile. In the lower plot there are residua of the fit. We notice, there are still some unaccounted peaks on the residua that may be due to low amplitude burst of emission. We are working on the method to distinguish between such bursts and the effects of statistical noise.

\section{Conclusions}

In the paper we have proposed the method of solar flares lightcurve parametrisation based on the soft X-rays observations. The elementary flare time profile was originally dedicated to analysis of flares observed by SphinX instrument. However, the formula is so general that it can be applied in the interpretation of other flaring lightcurves for another SXR solar observations as well. Presented approach assumes more realistic linear model of background, than just constant level. An appropriate estimation of background emission play the key role during any flares parameters estimation, especially in case of tiny events. Determined background emission itself can be used for the analysis of active regions parameters and their evolution.

Using proposed formula of elementary profile of simple flare allows for separation of time overlapped sequence of flare profiles which can be decomposed and analysed independently.

We have applied the method to interpretation of SphinX spectrophotometer lightcurve in order to systematic study distribution of flare characteristics observed during the minimum of activity. The proposed approach allow to create reliable catalogue of SphinX flares characteristic. Prepared database will be the subject of statistical analysis of flares recorded during the period of last solar minimum of activity.

\section{Acknowledgements}

The research leading to these results has been supported by Polish National Science Centre grant No. DEC-2013/09/N/ST9/02209.

\section{References}

Aschwanden, M. J., Dennis, B. R., \& Benz, A. O. 1998, ApJ., 497, 972.

Aschwanden, M. J. \& Freeland, S. L. 2012, ApJ, 754, 112.

Bornmann, P. L. 1990, ApJ, 356, 733

Bornmann, P. L., Speich, D., Hirman, J., Pizzo, V. J., Grubb, R., Balch, C., \& Heckman, G. 1996, SPIE Proc., 2812, 309.

Borucki, W. J., Koch, D., \& Basri, G., et al. 2010, Sci, 327, 977

Davenport, J. R. A., Hawley, S. L., Hebb, L., Wisniewski J. P., Kowalski, A., Johnson, E. C., Malatesta, M., Peraza, J., Keil, M., Silverberg, S. M., Jansen, T. C., Scheffler, M. S., Berdis, J. R., Larsen, D. M., \& Hilton E. J. 2014, ApJ, 797, 122

Fishman, G. J., Meegan, C. A., \& Wilson, R. B., et al. 1989, BAAS, 21, 860

Garcia, H. A. 1994, Solar Phys. 154, 275 
Gburek, S., Siarkowski, M., Kepa, A., Sylwester, J., Kowalinski, M., Bakala, J., Podgorski, P., Kordylewski, Z., Plocieniak, S., Sylwester, B., Trzebinski, W., \& Kuzin, S. 2011a, Solar System Research, 45, 182

Gburek, S., Sylwester, J., Kowalinski, M., Bakala, J., Kordylewski, Z., Podgorski, P., Plocieniak, S., Siarkowski, M., Sylwester, B., Trzebinski, W., Kuzin, S.V., Pertsov, A.A., Kotov, Y.D., Farnik, F., Reale, F., \& Phillips, K.J.H.2011b, Solar System Research, 45, 189.

Gburek, S., Sylwester, J., Kowalinski, M., Bakala, J., Kordylewski, Z., Podgorski, P., Plocieniak, S., Siarkowski, M., Sylwester, B., Trzebinski, W., Kuzin, S. V., Pertsov, A. A., Kotov, Y. D., Farnik, F., Reale, F., \& Phillips, K. J. H. 2013, Solar Phys., 283, 631

Markwardt, C. B. 2009, Non-linear Least-squares Fitting in IDL with MPFIT, 411, 251

More, J. J. 1977, The Levenberg-Marquardt algorithm: implementation and theory, 630, 105

Sylwester, J., Kowalinski, M., Gburek, S., Siarkowski, M., Kuzin, S., Farnik, F., Reale, F., Phillips, K. J. H., Baka la, J., Gryciuk, M., Podgorski, P., \& Sylwester, B. 2012, ApJ, 751, 111

Sylwester, J., Kuzin, S., Kotov, Y., Farnik, F.,\& Reale, F. 2008, ApJ (Letters), 29, 339

Sylwester, B., Sylwester, J., Siarkowski, M., Engell, A. J.,\& Kuzin, S. V. 2011, Central European Astrophysical Bulletin, 35, 171

Reinard, A., Hill, S., Viereck, R., \& Bailey, S. 2005, AGU Fall Meeting Abstracts, B344

van Beek, H. F., de Feiter, L. D., \& de Jager, C. 1974, In: Rycroft, M.J., Reasenberg, R.D. (eds.)

Space Research XIV, 447 